\title{
Integrated Vector Management on Mosquito Control During the COVID-19 Pandemic
}

\author{
Lutfhi Nurlaela*, Emma Mardliyah \\ Parasitology Department, Faculty of Medicine \\ Universitas Jenderal Achmad Yani \\ Bandung, Indonesia \\ *ufifuadi@gmail.com
}

\begin{abstract}
The coronavirus disease 2019 (COVID-19) pandemic caused by the severe acute respiratory syndrome coronavirus 2 (SARS-CoV-2) is now a major health crisis. In July 2020, there were 14 million cases and more than 600.000 deaths worldwide, creating great economic and social disruptions. Even though the pandemic is currently the main focus, plenty of other infectious diseases keep increasing. The World Health Organization (WHO) has previously highlighted the importance of preventing, detecting, and treating malaria amidst the current pandemic. A similar approach should be adopted for management of other arboviral diseases such as dengue, Zika, chikungunya, and yellow fever as was published in the PanAmerican Health Organization (PAHO)'s interim guideline regarding Aedes aegypti mosquito control during the COVID-19 pandemic. Public health interventions that were initiated to prevent COVID-19 spread immensely affect vector monitoring and control. A dengue or other vector borne disease (VBD) epidemic in addition to the current COVID-19 situation will have severe consequences for at risk populations. Cocirculation of dengue and COVID-19 may result in many unwanted outcomes such as co-infections; delayed diagnosis, management, and mitigation efforts; overwhelmed healthcare systems; less reported cases; less monitoring and control interventions; and exacerbation of social gaps. Clearly, the lockdown compromises vector control efforts, especially social mobilization campaigns and preventive insecticide spraying. Failure in implementing maximum vector control efforts may end up in reduced overall effectivity and increases risk of VBD circulation. The health community and policy makers should develop proactive efforts and allocate adequate resources in order to prevent and manage dengue and other VBDs in the current COVID-19 pandemic.
\end{abstract}

Keywords-integrated vector management, covid-19, vector borne diseases

\section{INTRODUCTION}

Dengue and other diseases carried by the Aedes mosquito (such as Chikungunya and Zika) affects 129 countries with more than 4 billion populations at risk worldwide. Dengue epidemics are usually seasonal, with transmissions peaking during and after rainy seasons. Dengue continues to circulate in several countries in 2020, with some countries even reporting an increased number of cases [1].
Cocirculation of dengue and coronavirus disease 2019 (COVID-19) may result in many unwanted outcomes such as co-infections; delayed diagnosis, management, and mitigation efforts; overwhelmed healthcare systems; less reported cases; less monitoring and control interventions; and exacerbation of social gaps. Clearly, the lockdown compromises vector control efforts, especially social mobilization campaigns and preventive insecticide spraying. Failure in implementing maximum vector control efforts may end up in reduced overall effectivity and increases risk of vector borne disease (VBD) circulation. The health community and policy makers should develop proactive efforts and allocate adequate resources in order to prevent and manage dengue and other VBDs in the current COVID-19 pandemic [2].

The emerging and re-emerging threat of VBDs is a huge health issue. More than half of the worldwide population is at risk for VBD, with morbidities exceeding one billion and mortalities exceeding one million annually. The World Health Organization (WHO) recommends an integrated vector management strategy (IVM) in order to reduce VBD transmission. IVM is defined as an approach taken in order to optimize resources for sustainable vector management with collaborations between health sectors, various public and private agents, and communities. It is made up of five key points: 1) evidence-based decision-making, 2) integrated approach, 3) collaboration within the health sector and with other sectors, 4) advocation, social mobilization, and legislation, and 5) capacity-building. In 2004 this strategy framework was adapted for all VBDs and is considered a rational approach in optimizing resources for vector control $[3,4]$.

IVM efforts use chemical and nonchemical methods, including environmental management. Effective and sustainable IVM is designed to achieve the best disease control with the most cost-effective method. Its application requires good understanding of the local vector and ecological transmission, and should be followed by the development of adequate infrastructure, funds, and human resources to manage and apply the program nationally and locally $[3,4]$ 


\section{CONSIDERATIONS AND RECOMMENDATIONS}

Simultaneous spread of COVID-19 and dengue may cause delayed diagnosis and poorly affect management, treatment, and interventions of each disease. A differential diagnosis is needed as both of these viral infections have similar clinical manifestations including fever, fatigue, and headache. In addition to that, there is concern of finding false positive serologic dengue cases in individuals with COVID-19. Confusion in diagnosis or in test results not only have severe consequences for the patient, but for public health control interventions (contact tracing in COVID-19 cases and vector control in dengue cases). Although co-infections of dengue and COVID-19 so far have only been sporadically reported in Thailand, Singapore, Mayotte, and the Reunion Island, it is a serious concern in countries experiencing dengue outbreaks, especially in Southeast Asia (including Indonesia) and South America. Healthcare facilities in most countries are disrupted or overwhelmed due to the COVID-19 pandemic, and the addition of VBDs will only worsen the situation. This is dangerous especially in areas where several dengue serotypes circulate and where secondary, even tertiary infections have been reported. Epidemiologic surveillance of dengue has also been affected. Currently in France there are less reports of dengue cases after lockdown, even though in the weeks prior there was a reported increase in cases. Lack of dengue reports may be due to not only the lockdown, but also the public's concern of COVID-19 transmission in healthcare facilities, the closure of several clinics, and disruption of diagnostic access [2].

After the start of the pandemic and the implementation of lockdowns, vector control interventions were scaled down, social mobilization campaigns were postponed, and preventive insecticide spraying were limited, especially in private spaces (Table1). The lack of vaccine and therapeutic management for dengue and other viral Aedes-borne diseases means that prevention and control of these diseases rely largely on vector population control or on terminating the human-vector contact through integrated, continuous, synergic, and proactive vector management. Failure in implementing vector control interventions may reduce its effectivity. In reality, many essential dengue control program interventions target private properties, like spraying residual insecticides inside spaces, door-to-door campaigns, eliminating sources, and residual peridomestic spraying. These interventions contradict the COVID-19 prevention guidelines and lockdown, as they require or will cause close contact between the vector control team and the public. Thus, key interventions of efficient dengue control are lessened and scaled down [2].

In order to reduce this gap, and while considering the effectivity of social mobilizations in control and prevention of Aedes-borne diseases, it is important to strengthen communication for social mobilization in fighting these diseases through digital communications and social media together with information regarding COVID-19, and to adapt existing guidelines to fit the current situation. Vector control workers should use personal protective equipment and maintain distances when conducting vector control interventions, including those that target larvae and adult mosquitoes, and community-based control efforts [2].

Negative effects of the COVID-19 crisis on dengue surveillance and control is apparent in tropical areas that are often affected by dengue outbreaks, but risks of local dengue and other arbovirus cases are expected to be lower in medium climate areas as limited travels reduce chances of virus importations. But the risk is still there, taking into account that 14 dengue cases were imported to France, Europe during the lockdown period from March 17 to May 11 2020, even though the number of flights from dengue affected areas were few. More air travels will bring more risks of viruses carried from arbovirus affected areas [2]

\section{BASIC GUIDELINES FOR STRATEGIC EFFORTS}

Each area is encouraged to use their resources effectively as many are allocated for COVID-19 response. This can be achieved by combining the following activities:

\section{A. Integrated Surveillance}

Countries are encouraged to continue their epidemiologic surveillances and provide reports from suspected cases and confirmed dengue cases. As clustered cases often occur in both dengue and COVID-19, surveillance and differential diagnosis efforts during laboratory examinations in each area need to be strengthened. Previously known dengue hotspots should be targeted for intensive vector control. Established clinical surveillance networks for dengue can widen their detection criteria to also include COVID-19 symptoms, in order to maximize resources. Sentinel entomologic surveillance will help assess changes in vector disease risk, and the impact of vector control measures [5].

\section{B. Diagnostics}

Many COVID-19 and dengue diagnostic examinations are PCR-based, but they use different reagents and sample types (oropharyngeal/nasopharyngeal swab for COVID-19 and blood/serum for dengue). In serologic test results, both virus aren't antigenetically related (they belong to different and distant viral families), so cross reactions are unlikely. In malaria co-endemic areas blood smear tests should be conducted in order to exclude the presence of malaria in the patient. Rapid diagnostic tests (RDT) are proven to have low sensitivity so the negative predictive value is very low, and a negative result cannot exclude a case. Because of this, implementation and use of this examination for public health needs must be properly considered, and ELISA should be prioritized for serology. Even though there is one report of a false positive dengue RDT in two COVID-19 cases, there is no proof to support antibody cross reactivity. Efforts are needed to improve virus surveillance and diagnostic capacity of laboratories in all countries [5]. 
TABLE I. EFFECTIVITY, STRENGTH OF EVIDENCE FROM VECTOR CONTROL EFFORTS, COVID-19 TRANSMISSION RISK ASSESSMENT IN VECTOR CONTROL WORKERS, AND RECOMMENDATIONS IN MAINTAINING VECTOR CONTROL EFFORTS IN LINE WITH COVID-19 PREVENTION [2].

\begin{tabular}{|c|c|c|c|c|c|}
\hline Vector control effort & Effectivity & $\begin{array}{c}\text { Strength of } \\
\text { evidence }\end{array}$ & $\begin{array}{l}\text { Type of COVID-19 } \\
\text { transmission risk }\end{array}$ & $\begin{array}{c}\text { Degree of } \\
\text { transmission risk }\end{array}$ & Recommendation \\
\hline \multicolumn{6}{|c|}{ Interventions in public areas } \\
\hline \multirow{2}{*}{ 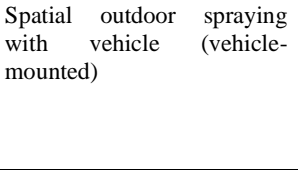 } & \multirow[t]{2}{*}{ Low } & \multirow[t]{2}{*}{ Low } & $\begin{array}{l}\text { Contact with other workers } \\
\text { in the same vehicle }\end{array}$ & Low & $\begin{array}{l}\text { One person per vehicle or distanced seating with masks } \\
\text { Vehicle ventilation (between each use) }\end{array}$ \\
\hline & & & $\begin{array}{l}\text { Contact with fomite in the } \\
\text { vehicle }\end{array}$ & Low & $\begin{array}{l}\text { One driver per journey } \\
\text { Hand washing } \\
\text { Regularly cleaning touched surfaces } \\
\text { Applying vehicle cleaning protocol after each use }\end{array}$ \\
\hline \multirow[t]{2}{*}{$\begin{array}{l}\text { Spatial outdoor spraying } \\
\text { with portable device }\end{array}$} & \multirow[t]{2}{*}{ Medium } & \multirow[t]{2}{*}{ Low } & Contact with public & Medium & $\begin{array}{l}\text { Distancing }(>1 \mathrm{~m}) \\
\text { Using masks/face shields } \\
\text { Reduce contact time with other people }\end{array}$ \\
\hline & & & Contact with families & Low & $\begin{array}{l}\text { Hand washing } \\
\text { Using disposable gloves }\end{array}$ \\
\hline \multirow[t]{2}{*}{$\begin{array}{l}\text { Eliminating non-permanent } \\
\text { breeding areas }\end{array}$} & \multirow[t]{2}{*}{ High } & \multirow[t]{2}{*}{ Medium } & Contact with public & Medium & $\begin{array}{l}\text { Distancing }(>1 \mathrm{~m}) \\
\text { Using masks/face shields } \\
\text { Reduce contact time with other people } \\
\text { Elimination should be done without interacting with residents }\end{array}$ \\
\hline & & & Contact with fomites & Medium & $\begin{array}{l}\text { Hand washing } \\
\text { Using disposable gloves }\end{array}$ \\
\hline \multirow[t]{2}{*}{ Larvicide } & \multirow[t]{2}{*}{ High } & \multirow[t]{2}{*}{ Medium } & Contact with public & Medium & $\begin{array}{l}\text { Distancing }(>1 \mathrm{~m}) \\
\text { Using masks/face shields } \\
\text { Reduce contact time with other people }\end{array}$ \\
\hline & & & Contact with fomite & Low & $\begin{array}{l}\text { Hand washing } \\
\text { Using disposable gloves }\end{array}$ \\
\hline \multicolumn{6}{|c|}{ Interventions in private spaces (households) } \\
\hline \multirow[t]{2}{*}{$\begin{array}{l}\text { Eliminating non-permanent } \\
\text { breeding areas }\end{array}$} & \multirow[t]{2}{*}{ Medium } & \multirow[t]{2}{*}{ Low } & Contact with residents & High & $\begin{array}{l}\text { Distancing }(>1 \mathrm{~m}) \\
\text { Using masks/face shields } \\
\text { Reduce contact time with other people } \\
\text { Elimination should be done without interacting with residents }\end{array}$ \\
\hline & & & Contact with fomite & High & $\begin{array}{l}\text { Hand washing } \\
\text { Using disposable gloves }\end{array}$ \\
\hline \multicolumn{6}{|l|}{ Social mobilizations } \\
\hline $\begin{array}{l}\text { Social mobilization (media, } \\
\text { social media, etc.) }\end{array}$ & Medium & $\begin{array}{l}\text { Low to } \\
\text { medium }\end{array}$ & No contact & Null & $\begin{array}{l}\text { No limits } \\
\text { Communications with prevention messages for COVID-19 and } \\
\text { dengue (including vector control efforts) }\end{array}$ \\
\hline
\end{tabular}

\section{Case Management}

Efforts to ensure appropriate clinical management on a suspected dengue case needs to be prioritized with strict applications of infection prevention and control in the context of COVID-19 transmission [5].

\section{Vector Control}

Efforts to reduce sources or mosquito breeding areas and adult mosquitoes should be done in affected or at risk areas. During lockdown, residents should be asked to cooperate to not leave water puddles, reduce solid garbage, and cover all water containers in and around their houses as a weekly family activity. In areas that are not under lockdown, vector control efforts that target larvae and adult mosquitoes should be implemented and vector control workers should use personal protective equipment when doing their job and maintain a safe distance. At risk groups (elderly, pregnant women, babies, and those that are sick) are urged to use insect repellents. Additional vector control efforts such as larvicides, targeted indoor residual spraying, and indoor spraying may be used to control dengue outbreaks depending on the resources, capacity, and plans of action developed in the area. Spatial insecticide application (fogging) is needed to quickly eliminate the adult mosquito population and reduce dengue transmission. Targeted indoor spraying should be selectively done in Aedes aegypti rest spaces, such as under furniture and on dark and damp surfaces. Careful to not fumigate water containers that are used for cooking and drinking. This intervention is effective up to 4 months, and can be used in healthcare centers, schools, and places of worship [5].

\section{E. Involving Communities}

All efforts need support from the community, whether to prevent COVID-19 or dengue. Materials about both diseases can be shown through media (including social media) if possible during lockdown. In areas where schools and classes have resumed, special sessions should be held to increase awareness on COVID-19 and dengue prevention. Media campaigns can be continued for dengue prevention and Aedes mosquito control [5].

\section{CONCLUSION}

As previously mentioned, the importance of maintaining and improving integrated VBD management needs to be highlighted, especially surveillance and vector control in private spaces and social mobilization during the COVID-19 pandemic, and the importance of improving guidelines to include reducing the workers and the public's exposure to risks. Although several countries have already started to lift lockdowns, there are still concerns regarding the possibility of 
a second wave of COVID-19 epidemic, and new lockdowns (strict or partial) are being done in a few areas.

All around the world we are facing an imminent potential risk of arboviral disease such as dengue fever, chikungunya, Zika, and yellow fever simultaneously emerging due to reduced vector control and surveillance during the COVID-19 pandemic, resulting in an even worse situation. We would like to encourage the health community and policy makers to immediately develop proactive policies and to allocate adequate resources in order to prevent and manage the reemergence of VBDs during the COVID-19 pandemic. On a larger scale, in the context of risks for new disease emerging with high epidemic and pandemic potential, preparation is essential to maintain and strengthen VBD control activities.

\section{REFERENCES}

[1] Pan American Health Organization, "Dengue Prevention and Control during COVID-19 Pandemic," PAHO, 2020

[2] M.-M. Olive, T. Baldet, J. Devillers, J. Fite, M.-C. Paty, C. Paupy, P. Quénel, E. Quillery, J. Raude, J.-P. Stahl, M. Thiann-Bo-Morel, and D. Roiz, "The COVID-19 Pandemic Should Not Jeopardize Dengue Control.," PLoS Negl. Trop. Dis., vol. 14, no. 9, p. e0008716, 2020.

[3] E. Chanda, B. Ameneshewa, M. Bagayoko, J.M. Govere, and M.B. Macdonald, "Harnessing Integrated Vector Management for Enhanced Disease Prevention.," Trends Parasitol., vol. 33, no. 1, pp. 30-41, 2017.

[4] J.C. Beier, J. Keating, J.I. Githure, M.B. Macdonald, D.E. Impoinvil, and R.J. Novak, "Integrated Vector Management for Malaria Control.,' Malar. J., vol. 7 Suppl 1, no. Suppl 1, p. S4, 2008.

[5] F. Seelig et al., "The COVID-19 Pandemic Should Not Derail Global Vector Control Efforts," PLoS Negl. Trop. Dis., vol. 14, no. 8, p. e0008606, 2020. 